\title{
Spaceborne Demonstration of Distributed SAR Imaging with TerraSAR-X and TanDEM-X
}

\author{
Thomas Kraus, Gerhard Krieger, Markus Bachmann, and Alberto Moreira
}

\begin{abstract}
Multistatic or distributed satellite systems offer new and unique capabilities necessary for Earth observation with high spatial and temporal resolution. This letter describes a multistatic synthetic aperture radar (SAR) experiment employing the satellites TerraSAR-X and TanDEM-X. In order to demonstrate distributed SAR imaging from space, special data acquisitions with a dedicated geometry were performed. The data evaluation approach is outlined and azimuth profiles over a region with high-contrast backscatter are used to evaluate the azimuth signal reconstruction performance. The results are verified with simulations performed with a flexible SAR simulator reproducing the acquired scene. Finally, the effect of target motion on the reconstruction is analyzed and discussed based on the experimental data.
\end{abstract}

Index Terms-TerraSAR-X, TanDEM-X, high resolution wide swath, multistatic, distributed satellite systems, synthetic aperture radar (SAR).

\section{INTRODUCTION}

$\mathbf{T}$ HE aspiration to image very wide swaths with high azimuth resolution is limited by contradicting pulse repetition frequency (PRF) requirements. Wide swaths require long echo windows which in turn means a small PRF. To fulfill the sampling theorem in azimuth direction, however, a PRF larger than the Nyquist rate is necessary. Multichannel SAR imaging is a promising technique to overcome these contradictions of conventional single-channel SAR systems. Multichannel systems with a sampling approach as proposed in [1] offer the necessary degree of freedom to overcome these singlechannel sampling limitations. A generalized solution for the signal reconstruction under nonuniform sampling conditions is developed in [2]. The latter approach has been further elaborated and extended, e.g., in [3], [4], [5]. An early spaceborne demonstration of the technique using TerraSAR-X data is presented in [6]. Further spaceborne results were reported in [4]. However, the concept of multichannel azimuth signal reconstruction is not limited to single-platform systems. It is even more promising for bi- and multistatic SAR systems. The vision of a constellation of SAR satellites, enabling at the same time high-resolution wide-swath SAR imaging and interferometric and tomographic applications, is outlined in [7]. The cross-platform idea is further elaborated in [8] and first experimental results are reported in [9] and [10]. This letter describes for the first time in detail the results of a distributed SAR imaging experiment performed with TerraSAR-X and TanDEM-X.

The authors are with the Microwaves and Radar Institute, German Aerospace Center (DLR), 82234 Oberpfaffenhofen, Germany (e-mail: T.Kraus@dlr.de).

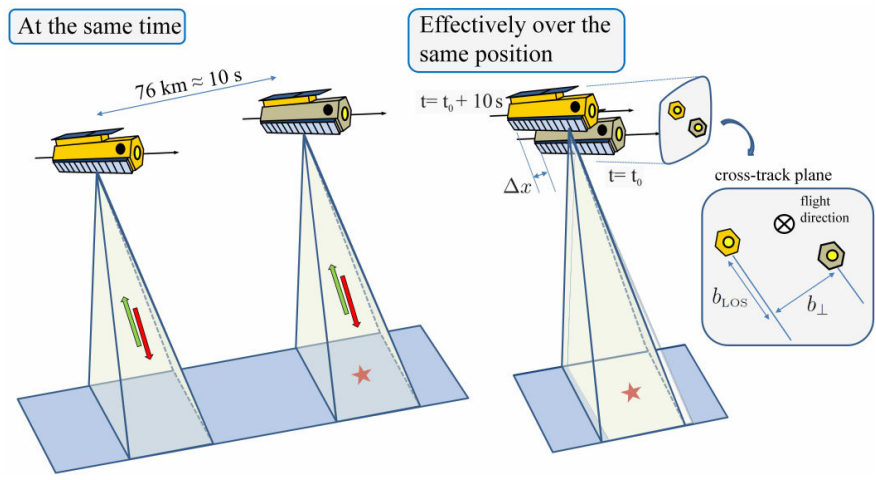

Fig. 1. Pursuit monostatic acquisition mode geometry depicted at a single instant of time $t_{0}$ (left) and over a point target on ground at the times $t_{0}$ and $t_{0}+10 \mathrm{~s}$ (right). The baselines in along-track $\Delta x$, perpendicular $b_{\perp}$, and line-of-sight $b_{\text {LOS }}$ direction are shown.

The paper is structured as follows. In section II the experimental acquisition is described. The simulation framework is introduced in section III. Section IV compares the results obtained from the experiment with simulation results. Additionally, the effect of target motion on the reconstructed signal is discussed. Section V concludes the paper.

\section{EXPERIMENTAL ACQUiSITION}

For the experiment dedicated data takes in stripmap mode have been acquired with TerraSAR-X and TanDEM-X. The acquisitions took place in December 2014. During this mission phase both spacecraft were flying in a pursuit monostatic formation with an along-track separation between TerraSAR-X and TanDEM-X of about ten seconds as shown in Fig. 1 on the left [11], [12], [13]. Both radars are operated independently but acquire the same scene. Despite the ten seconds separation, the resulting along-track baseline is in the order of meters. The along-track baseline only depends on the start of the individual acquisitions over the scene and the resulting spatial interleaving of the samples as shown in Fig. 1 on the right.

The cross-track baseline during this mission phase varied around the orbit according to the chosen helix parameters of the formation flight of the satellites. In this phase the cross-track baseline was very small over Antarctica. Therefore, the experimental acquisitions have been planned and ordered there. The resulting perpendicular cross-track baseline is in the order of one meter, the line-of-sight baseline is in the order of ten meters. The small interferometric baseline leads to a low sensitivity to the topography which eases the correction of the topographic phase. 

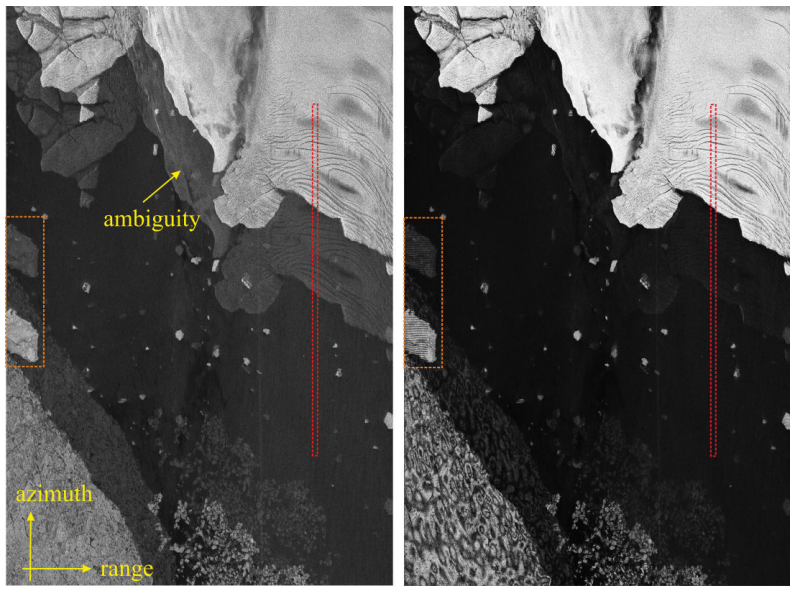

Fig. 2. The TerraSAR-X image (left) shows strong azimuth ambiguities. The reconstructed image (right) is based on the data of TerraSAR-X and TanDEM-X (right). The reconstructed image is processed with an azimuth bandwidth of one PRF, i.e., $2000 \mathrm{~Hz}$ in order to achieve the same spatial resolution as the single-channel image. The red rectangles mark the area where the power profiles shown in Fig. 5 are generated from. The orange rectangles highlight a floating ice floe and one of its ambiguities (cf. section IV-C).

The PRFs of the SAR instruments were adjusted to lower than nominal values in order to provoke azimuth ambiguities in the single-channel SAR data. The goal is now to reduce these ambiguities by multistatic azimuth signal reconstruction. The actual scene was chosen to contain a high contrast region, e.g., a land-sea transition, where azimuth ambiguities are easily recognized as shown in Fig. 2 on the left. The image shows a part of the whole acquisition which has a total duration of $23 \mathrm{~s}$, corresponding to about $160 \mathrm{~km}$. The right image shows a two-channel reconstructed image with a lower ambiguity level. The improvement is clearly visible. A quantitative evaluation is presented in section IV-A.

In Fig. 3 the processing approach for the multichannel data is sketched for the general case of $N$ channels. The block diagram starts with the range focusing followed by a co-registration in range for all but the master channel to remove the line-of-sight baseline and range sampling time offsets between the channels. For the slave channels the cross-track baseline is compensated using either a flat earth model or a digital elevation model (DEM). As the cross-track baseline is small in the described experiment, the topography does not have a significant impact. Ideas about topography compensation algorithms for data which are sampled below the Nyquist rate are further elaborated in [14]. The impact of large along-track baselines is discussed in [15].

Before the multichannel reconstruction is conducted, the channels are balanced using a histogram based approach [16]. Gain and phase imbalances between the channels are a result of the used hardware. First, the along-track baseline related phase term is removed from the data. It is modeled as a linear phase ramp over the Doppler frequency. Then, the channels are adjusted in power and phase with respect to the master - the actual balancing. After this, the along-track baseline component is re-introduced. This preserves the along-track baseline related phase term. Otherwise the channel balancing would treat it as an error and remove it.

Instead of performing the reconstruction on raw data like in [6], it was decided to do it on range focused data. This is necessary as the flat earth component, and additionally a topographic variation within the uncompressed range pulse, would lead to non-corrected phase disturbances. For the experimental acquisition the pulse length is $67 \mu$ s which equals $20 \mathrm{~km}$ in slant range. Over this range the phase used to correct the topography significantly changes. As multichannel systems seek to use lower values of the PRF to enable wide-swath imaging, the length of the used pulses is even higher, as the number of receivers increases. This confirms the necessity to perform the range focusing very early in the processing chain.

The signal reconstruction assumes the channel transfer function of the $i$-th channel as described in [2],

$$
H_{i}(f)=\exp \left(-\mathrm{j} \frac{\pi \Delta x_{i}^{2}}{2 \lambda r_{0}}\right) \cdot \exp \left(-\mathrm{j} 2 \pi \frac{\Delta x_{i}}{2 v} f\right)
$$

where $\Delta x_{i}$ denotes the along-track baseline, $\lambda$ the wavelength, $r_{0}$ the slant range, $v$ the platform velocity and $f$ the Doppler frequency. Finally, the azimuth focusing including range cell migration correction is performed. The basis for the processing environment is the TAXI processor (experimental TanDEM-X interferometric processor) [17] which was specifically adapted and extended for this evaluation. Compared to [6] the coregistration in range direction and the correction of the topographic phase are key features which have been introduced into the processing chain in order to take the cross-track baseline into account.

As a further simplification, the time-variance of the alongtrack baseline is neglected. This assumption is valid as long as the variation is slow and the considered part of the acquisition is short. Both conditions are fulfilled for the analyzed data set shown here.

\section{Simulation FramewORK}

The multistatic simulation framework is based on a point target simulator. It is implemented with very flexible input parameters. A block diagram is shown in Fig. 4. The inputs are on one side the system parameters like the number of apertures, the aperture sizes, the PRF, the signal-to-noise ratio (SNR), and other processing parameters like the processed azimuth bandwidth. On the other side, the geometry of the data acquisition is parametrized by the sensor's trajectories, velocities and attitudes. These input parameters are chosen to match with a certain real acquisition to compare the simulated performance results with measured ones. They can also be set to predefined values in order to estimate the performance of a possible future multistatic SAR mission. The actual orbit and attitude data of the satellites are imported into the simulation and a flat earth model is used. For the antenna patterns, the TerraSAR-X antenna patterns are used [18]. The core part of the simulation framework consists of the generation of the signals and the implementation of the azimuth signal reconstruction as described in [2] or [4]. In a subsequent step, the reconstructed signal is analyzed regarding different parameters, e.g., the azimuth ambiguity-to-signal ratio (AASR), 


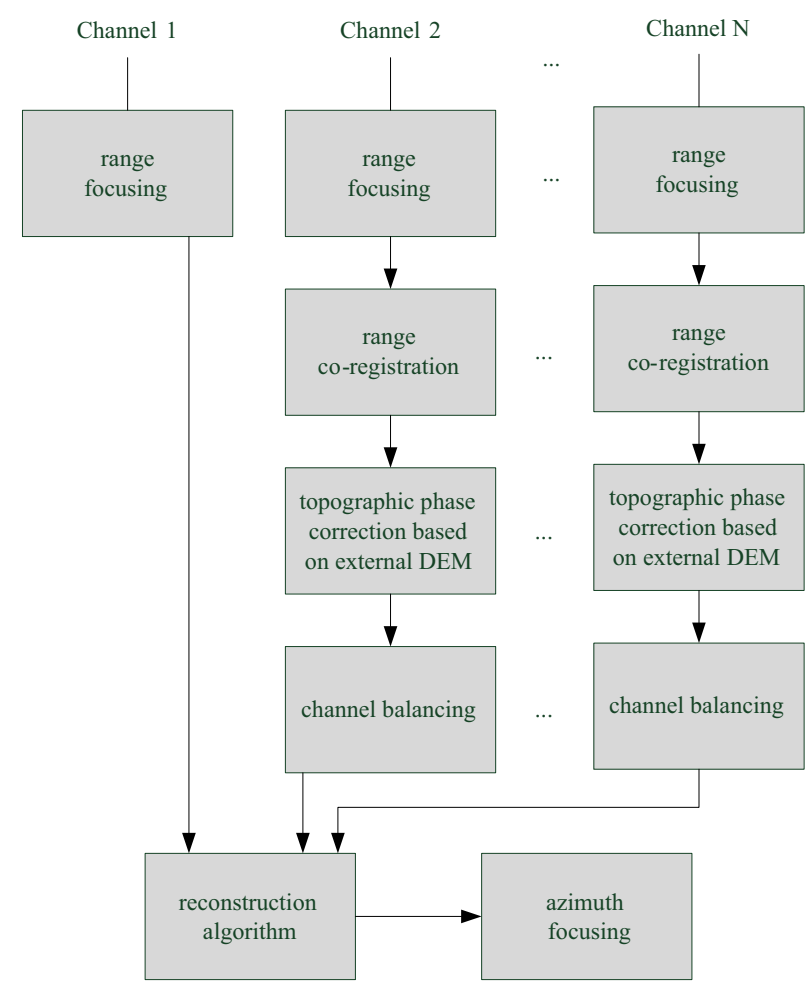

Fig. 3. Block diagram of the azimuth signal reconstruction processing.

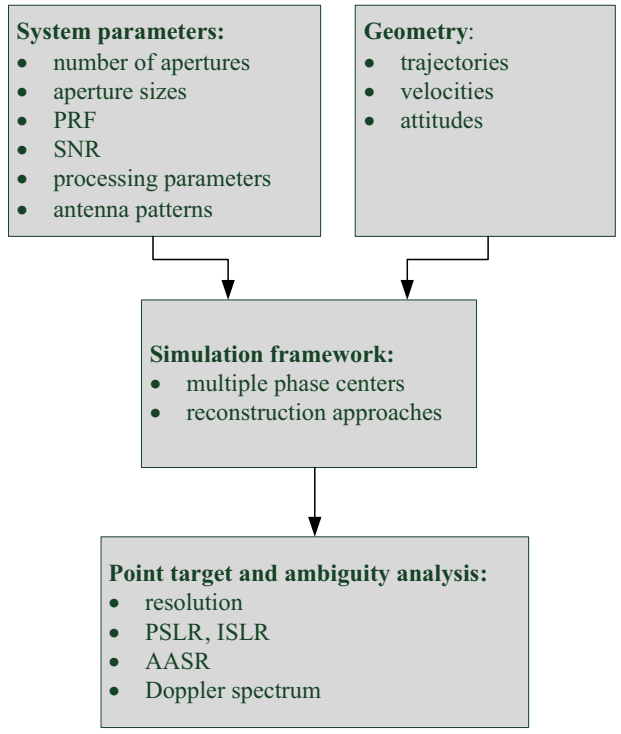

Fig. 4. Block diagram of the simulation framework used to simulate the multistatic azimuth signal reconstruction. PSLR stands for peak sidelobe ratio, ISLR for integrated sidelobe ratio, and AASR for azimuth ambiguity-to-signal ratio.

impulse response function (IRF) parameters like resolution and side-lobe levels, or the shape of the Doppler spectrum.

\section{Data Analysis}

In this section, results from the experimental acquisition are shown and compared to simulation results. Finally, an effect detected in the data is analyzed.

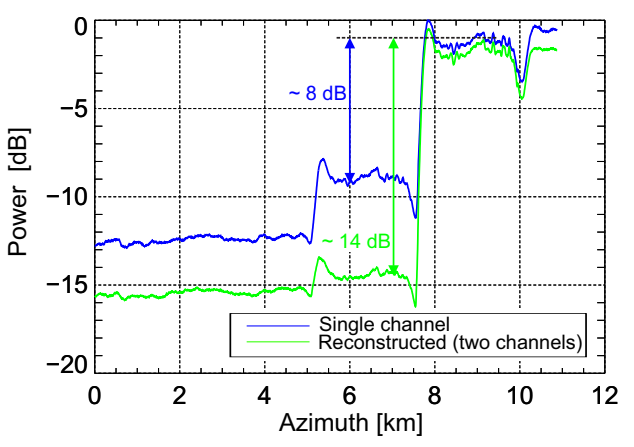

Fig. 5. Azimuth profiles along the sea-land transition (red rectangle in Fig. 2) for the single-channel TerraSAR-X dataset in blue and for the reconstructed dataset based on TerraSAR- $X$ and TanDEM- $X$ data in green.

\section{A. Experimental Results}

In order to evaluate the AASR performance of the actual images, an azimuth profile along a high contrast region can be analyzed as shown by the red rectangles in Fig. 2. The normalized power profile of the single-channel TerraSAR-X image is shown in blue in Fig. 5. The green curve represents the power profile of the two-channel reconstructed data. For comparability reasons both images have been processed to the same azimuth resolution. Therefore, a SNR gain of about $3 \mathrm{~dB}$ is visible in the azimuth range between 0 and $5.2 \mathrm{~km}$ for the two-channel data. In this area the backscatter of the sea is very low and without ambiguities so that the received signal is dominated by the noise power.

The ambiguity power is clearly visible between 5.2 and $7.6 \mathrm{~km}$, especially for the single-channel profile. The distance $d_{\text {ambi }}$ of about $2.4 \mathrm{~km}$ between the actual target area and its ambiguity coincides with the predicted position, calculated from the PRF according to [19], [6]

$$
d_{\mathrm{ambi}} \approx \frac{f_{\mathrm{PRF}} \lambda r_{0} v_{\mathrm{g}}}{2 v_{\mathrm{eff}}^{2}},
$$

where $f_{\mathrm{PRF}}$ is the PRF, $v_{\mathrm{g}}$ and $v_{\text {eff }}$ are the ground the effective velocity, respectively.

The dominant ambiguity power originates from the area between 7.6 and $10.0 \mathrm{~km}$. Comparing the power levels, the resulting ambiguity suppression is about $-8 \mathrm{~dB}$ for the singlechannel profile and about $-14 \mathrm{~dB}$ for the two-channel reconstructed profile as visible in Fig. 5.

\section{B. Simulation Results}

The measurement results can now be compared to simulation results. In Fig. 6 the simulated AASR for an acquisition scenario similar to the actual experimental acquisition is shown as a function of the PRF. The blue curve represents the AASR of a single channel, the green one corresponds to the AASR after two-channel reconstruction. As expected, the AASR performance of the single-channel data improves as the PRF increases. The AASR of the reconstructed signal, however, improves until the PRF is $2.25 \mathrm{kHz}$ and after that it worsens. The reason for this behavior is the distribution of the spatial samples [2]. For the given geometry, a PRF of about $2.2 \mathrm{kHz}$ leads to a uniform sampling of the received signals 


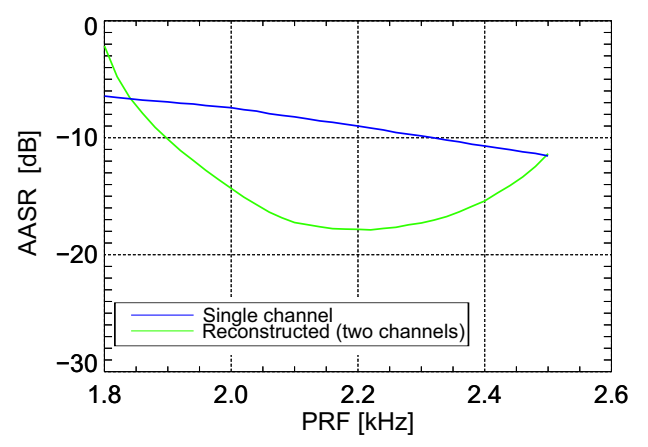

Fig. 6. Simulated AASR performance for the geometry and system parameters equivalent to the experimental satellite acquisitions as a function of the PRF. The imaging PRF of the actual acquisition was $2 \mathrm{kHz}$.

in the azimuth direction. Increasing the PRF further increases the non-uniformity of the sampling which compensates the benefit of a larger PRF and even further deteriorates the AASR performance. The PRF of the actual single-channel acquisition was $2 \mathrm{kHz}$ leading to a sampling uniformity of 0.6 for a scale between 0.0 and 2.0 (uniform sampling for 1.0) [20]. This results in a theoretical AASR performance of about $-14 \mathrm{~dB}$ for the reconstructed signal at the PRF of $2 \mathrm{kHz}$. The single channel achieves an AASR of about $-7.5 \mathrm{~dB}$. Thus, the simulation results correspond very well to the values derived from the azimuth profile shown in Fig. 5.

An indicator for a correct implementation of the reconstruction is the Doppler spectrum. By evaluating a highbackscatter area of the scene, a Doppler spectrum of the reconstructed signal can be derived. The spectrum of the twochannel reconstructed signal is shown in blue in Fig. 7. The red curve is derived using the simulation framework. The input parameters of the simulation like the PRF, the separation of the apertures, and the processing parameters are set in accordance with the values of the actual acquisition. Both spectra fit very well, indicating a successful reconstruction. In order to highlight the effect of the reconstruction, the spectrum of a single channel is shown in orange. Due to severe aliasing, this spectrum is almost flat. For comparison the azimuth antenna pattern is shown in green. It can serve as a rough hint of what the reconstructed Doppler spectrum should look like. However, it does not take into account residual ambiguities from outside the reconstructed azimuth frequency band, as those ambiguities are not received via the shown part of the antenna pattern. Additionally, an imperfect ambiguity suppression as well as the impact of noise leads to differences between the spectrum and the antenna pattern shape. Therefore, the difference between the antenna pattern and the reconstructed Doppler spectrum is a very valuable indicator for imperfections as well as the inherent limitations of the proposed reconstruction. The simulated spectrum can be used to estimate the performance of future multistatic SAR systems.

\section{Analysis of Ambiguity Suppression}

In Fig. 2 an ice floe with one of its ambiguities is highlighted by the orange box. The other ambiguity is overlaid by floating

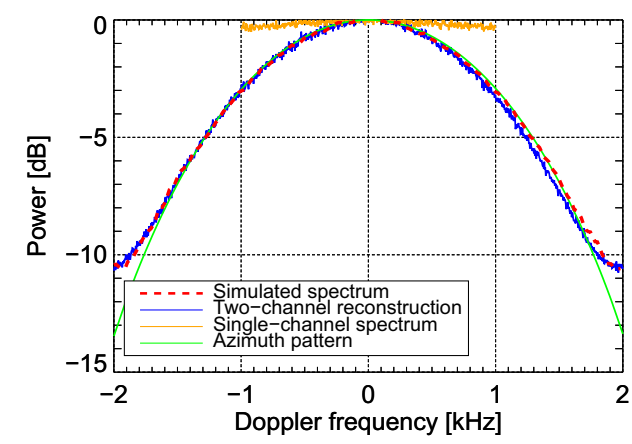

Fig. 7. Simulated Doppler spectrum (red), Doppler spectrum of the reconstructed image (blue), single-channel Doppler spectrum (orange), and the azimuth antenna pattern of TerraSAR-X as reference (green).

ice masses on the lower part of the image and is therefore not recognizable. Obviously, in the right image the ambiguity of the ice floe is not well suppressed, compared to the ambiguities of the solid ice mass at the coast. Additionally, a slight amplitude modulation in azimuth direction in the reconstructed image, both for the ice floe and its ambiguity is visible. To highlight this phenomenon another ice floe is depicted in Fig. 8. This sub-image is part of the same acquisition close to Antarctica but further north. The amplitude-modulation-like phenomenon in the reconstructed image here is clearly visible but has a different frequency. This behavior is very likely related to a rotation of the ice floe during the approximately ten seconds time-lag between the TerraSAR-X and the TanDEM-X acquisition. For interferometric applications, a similar feature is reported for the interferometric phase when ice floes are observed [21], [22], [23]. There, the presence of along-track fringes in the interferograms is explained by a rotation of the ice floes around their vertical axes.

In contrast to the single solid ice floe presented in Fig. 8, the ice structure in the lower left corner of Fig. 2 consists of many small individual ice floes. Therefore, the azimuth phase pattern visible for the larger solid ice floe is not recognizable. Nevertheless, a degraded ambiguity cancellation is also visible in the right image.

If a two-channel azimuth signal reconstruction is applied, the additional phase component results in constructive and destructive interference in along-track direction. During the reconstruction, the phase of the slave channel is expected to have a certain behavior with respect to the master channel according to the azimuth baseline or more general the observation geometry, cf. Eq. 1. However, due to the rotation of the ice floe in the time-lag between both acquisitions, an additional azimuth variant phase term is introduced, disturbing the reconstruction. The additional phase term prohibits constructive summing of the signal for the actual target as well as a destructive interference for the ambiguities depending on the azimuth position.

On the one hand, this effect is a challenge concerning multichannel signal reconstruction and azimuth ambiguity suppression. But on the other hand, it can also be seen as a chance for moving object detection. In [22] it has been shown that the rotation of the ice floes can be estimated with 


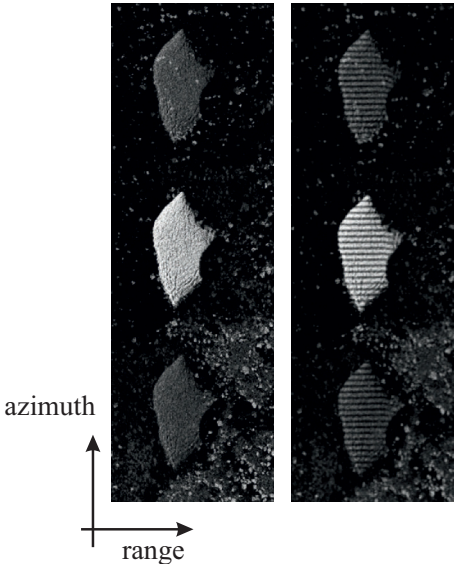

Fig. 8. Another part of the experimental TerraSAR-X acquisition showing a ice floe with both azimuth ambiguities (left). The reconstructed multistatic image based on the data of both satellites shows a characteristic amplitude modulation in azimuth direction, both for the actual ice floe as well as the ambiguities (right).

millidegree accuracy. By means of a proper phase correction, a modulation-free image can be potentially obtained.

\section{CONCLUSION AND Discussion}

This letter presents a distributed SAR imaging experiment employing TerraSAR-X and TanDEM-X. The data evaluation approach is outlined and the ambiguity performance of the reconstructed image is compared to simulation results, with very good agreement.

The necessity to perform the range focusing early in the processing chain is highlighted and challenges during the topography compensation are discussed.

Additionally, the effect of an azimuth amplitude modulation for certain targets is shown. The root cause for this behavior, occurring after the azimuth signal reconstruction is identified to be a rotation of the ice floes introducing a disturbing phase term. This effect caused by non stationary targets is not present for distributed SAR systems in close formation. However, for a constellation of $N$ monostatic SARs as, e.g., the SAR train proposed in [24], the effect has to be considered.

The presented results are promising. They are regarded as an important step towards the vision of distributed SAR systems. Nevertheless, many interesting questions remain to be analyzed, especially concerning the consideration of the topography for geometries with larger baselines as well as the time variance of the geometry. Multistatic reconstruction for reflector based SAR systems with several azimuth channels is also a topic for future research.

\section{REFERENCES}

[1] A. Currie and M. A. Brown, "Wide-swath SAR," in Proc. Inst. Elect. Eng.-Radar, Sonar, Navigat., vol. 139, no. 2, 1992, pp. 122-135.

[2] G. Krieger, N. Gebert, and A. Moreira, "Unambiguous SAR signal reconstruction from nonuniform displaced phase center sampling," IEEE Geosci. Remote Sens. Lett., vol. 1, no. 4, pp. 260-264, 2004.

[3] N. Gebert, G. Krieger, and A. Moreira, "Digital beamforming on receive: Techniques and optimization strategies for high-resolution wide-swath SAR imaging," IEEE Trans. Aerosp. Electron. Syst., vol. 45, no. 2, pp. 564-592, 2009.
[4] D. Cerutti-Maori, I. Sikaneta, J. Klare, and C. Gierull, "MIMO SAR processing for multichannel high-resolution wide-swath radars," IEEE Trans. Geosci. Remote Sens., vol. 52, no. 8, pp. 5034-5055, 2014.

[5] I. Sikaneta, C. Gierull, and D. Cerutti-Maori, "Optimum signal processing for multichannel SAR: With application to high-resolution wideswath imaging," IEEE Trans. Geosci. Remote Sens., vol. 52, no. 10, pp. 6095-6109, 2014.

[6] J.-H. Kim, M. Younis, P. Prats-Iraola, M. Gabele, and G. Krieger, "First spaceborne demonstration of digital beamforming for azimuth ambiguity suppression," IEEE Trans. Geosci. Remote Sens., vol. 51, no. 1, pp. 579590, 2013.

[7] G. Krieger and A. Moreira, "Spaceborne bi- and multistatic SAR: potential and challenges," Proc. Inst. Elect. Eng.-Radar, Sonar, Navigat., vol. 153, no. 3, pp. 184-198, 2006.

[8] M. Rodriguez-Cassola, P. Prats-Iraola, U. Steinbrecher, D. Schulze, G. Krieger, A. Reigber, and A. Moreira, "Cross-platform spaceborne SAR imaging: Demonstration using TanDEM-X," in Proc. IGARSS, 2013, pp. 2962-2965.

[9] T. Kraus, B. Bräutigam, M. Bachmann, and G. Krieger, "Multistatic SAR imaging: First results of a four phase center experiment with TerraSAR-X and TanDEM-X," in Proc. EUSAR, Hamburg, Germany, 2016.

[10] T. Kraus, M. Bachmann, L. Heiderich, G. Krieger, and A. Moreira, "Multistatic SAR imaging: Comparison of simulation results and experimental data," in Proc. Inst. Elect. Eng.-Radar, Sonar, Navigat. IET, 2017.

[11] G. Krieger, A. Moreira, H. Fiedler, I. Hajnsek, M. Werner, M. Younis, and M. Zink, "TanDEM-X: A satellite formation for high-resolution SAR interferometry," IEEE Trans. Geosci. Remote Sens., vol. 45, no. 11, pp. 3317-3341, 2007.

[12] G. Krieger, M. Zink, M. Bachmann, B. Bräutigam, D. Schulze, M. Martone, P. Rizzoli, U. Steinbrecher, J. W. Antony, F. De Zan, I. Hajnsek, K. Papathanassiou, F. Kugler, M. Rodriguez Cassola, M. Younis, S. Baumgartner, P. López-Dekker, and A. Moreira, "TanDEM-X: A radar interferometer with two formation-flying satellites," Acta Astronautica, vol. 89, pp. 83-98, 2013.

[13] M. Zink, M. Bachmann, B. Bräutigam, T. Fritz, I. Hajnsek, A. Moreira, B. Wessel, and G. Krieger, "TanDEM-X: The new global DEM takes shape," IEEE Geosci. Remote Sens. Mag., vol. 2, pp. 8-23, 2014.

[14] T. Kraus, M. Bachmann, and G. Krieger, "Topography correction for distributed SAR imaging: A case study for TerraSAR-X and TanDEM-X," in Proc. EUSAR, 2018.

[15] N. Sakar, M. Rodriguez-Cassola, P. Prats-Iraola, A. Reigber, and A. Moreira, "Analysis of geometrical approximations in signal reconstruction methods for multistatic SAR constellations with large along-track baseline," IEEE Geosci. Remote Sens. Lett., vol. 15, pp. 892-896, 2018.

[16] C. H. Gierull, "Digital channel balancing of along-track interferometric SAR data," Defence R\&D Canada-Ottawa, Tech. Rep., 2003.

[17] P. Prats, M. Rodriguez-Cassola, L. Marotti, M. Naninni, S. Wollstadt, D. Schulze, N. Tous-Ramon, M. Younis, G. Krieger, and A. Reigber, "TAXI: A versatile processing chain for experimental TanDEM-X product evaluation," in Proc. IGARSS, 2010, pp. 4059-4062.

[18] M. Bachmann, M. Schwerdt, and B. Bräutigam, "TerraSAR-X antenna calibration and monitoring based on a precise antenna model," IEEE Trans. Geosci. Remote Sens., vol. 48, no. 2, pp. 690-701, 2010.

[19] J. Curlander and R. McDonough, Synthetic Aperture Radar: Systems and Signal Processing. Wiley, 1991.

[20] M. Kiemer and H. Breit, "Efficient evaluation of multichannel SAR data recombination filters," IEEE Trans. Geosci. Remote Sens., vol. 55, no. 11, pp. 6277-6286, 2017.

[21] M. Thomas, M. Steffens, and R. Roth, "Measuring changes of iceberg attitudes by SAR interferometry," in Third ERS Symposium on Space at the service of our Environment, vol. 414, 1997, p. 955.

[22] R. Scheiber, F. D. Zan, P. Prats, L. S. Araújo, M. Künemund, and L. Marotti, "Interferometric sea ice mapping with TanDEM-X: First experiments," in Proc. IGARSS, 2011.

[23] P. López-Dekker, P. Prats, F. De Zan, D. Schulze, G. Krieger, and A. Moreira, "TanDEM-X first DEM acquisition: a crossing orbit experiment," IEEE Geosci. Remote Sens. Lett., vol. 8, no. 5, pp. 943-947, 2011.

[24] J. P. Aguttes, "The SAR train concept: An along-track formation of SAR satellites for diluting the antenna area over $n$ smaller satellites, while increasing performance by n," Acta Astronautica, vol. 57, no. 2, pp. 197 $-204,2005$. 